\title{
Metallothionein alleviates cardiac contractile dysfunction induced by insulin resistance: role of Akt phosphorylation, PTB1B, PPARY and c-Jun
}

Received: 5 January 2005 / Accepted: 17 June 2005 / Published online: 20 September 2005

(C) Springer-Verlag 2005

\begin{abstract}
Aims/hypothesis: Insulin resistance is concomitant with metabolic syndrome, oxidative stress and cardiac contractile dysfunction. However, the causal relationship between oxidative stress and cardiac dysfunction is unknown. This study was designed to determine the impact of overexpression of the cardiac antioxidant metallothionein on cardiac dysfunction induced by insulin resistance in mice. Methods: Whole-body insulin resistance was generated in wild-type FVB and metallothionein transgenic mice by feeding them with sucrose for 12 weeks. Contractile and intracellular $\mathrm{Ca}^{2+}$ properties were evaluated in ventricular myocytes using an IonOptix system. The contractile indices analysed included: peak shortening (PS), time to 90\% PS ( $\mathrm{TPS}_{90}$ ), time to $90 \%$ relengthening $\left(\mathrm{TR}_{90}\right)$, halfwidth duration, maximal velocity of shortening $(+\mathrm{d} L / \mathrm{d} t)$ and relengthening $(-\mathrm{d} L / \mathrm{d} t)$, fura-fluorescence intensity change $(\Delta \mathrm{FFI})$ and decay rate $(\tau)$. Results: The sucrose-fed mice displayed glucose intolerance, enhanced oxidative stress, hyperinsulinaemia, hypertriglyceridaemia and normal body weight. Compared with myocytes in starch-fed mice, those from sucrose-fed mice exhibited depressed PS, $+\mathrm{d} L / \mathrm{d} t,-\mathrm{d} L /$ $\mathrm{d} t$, prolonged $\mathrm{TR}_{90}$ and decay rate, and reduced $\Delta \mathrm{FFI}$ associated with normal $\mathrm{TPS}_{90}$ and half-width duration. Western blot analysis revealed enhanced basal, but blunted insulin $(15 \mathrm{mU} / \mathrm{g})$-stimulated Akt phosphorylation. It also showed elevated expression of insulin receptor $\beta$, insulin receptor tyrosine phosphorylation, peroxisome proliferatoractivated receptor $\gamma$, protein tyrosine phosphatase 1B and
\end{abstract}

C. X. Fang $\cdot$ F. Dong $\cdot$ B. H. Ren · J. Ren $(\bowtie)$

Division of Pharmaceutical Sciences \& Center

for Cardiovascular Research and Alternative Medicine, University of Wyoming,

Laramie, WY, 82071-3375, USA

e-mail: jren@uwyo.edu

Tel.: +1-307-7666131

Fax: +1-307-7662953

P. N. Epstein

Department of Pediatrics,

University of Louisville,

Louisville, KY, USA phosphorylation of the transcription factor c-Jun, associated with a reduced fold increase of insulin-stimulated insulin receptor tyrosine phosphorylation in sucrose-fed mice. All western blot findings may be attenuated or ablated by metallothionein. Conclusions/interpretation: These data indicate that oxidative stress may play an important role in cardiac contractile dysfunction associated with glucose intolerance and possibly related to alteration in insulin signalling at the receptor and post-receptor levels.

Keywords Antioxidant · Insulin resistance · Intracellular $\mathrm{Ca}^{2+}$ transients $\cdot$ Myocyte $\cdot$ Shortening

Abbreviations $+\mathrm{d} L / \mathrm{d} t$ : maximal velocity of shortening . $-\mathrm{d} L / \mathrm{d} t$ : maximal velocity of relengthening $\cdot$ FFI: fura- 2

fluorescence intensity - GSH: glutathione - GSSG: oxidised glutathione - MT: metallothionein - PI-3K:

phosphatidylinositol-3 kinase $\cdot$ PPAR $\gamma$ : peroxisome

proliferator-activated receptor $\gamma$. PS: peak shortening .

PTP1B: protein tyrosine phosphatase 1B - TPS $_{90}$ : time to $90 \%$ peak shortening $\cdot \mathrm{TR}_{90}$ : time to $90 \%$ relengthening

\section{Introduction}

Insulin resistance is a hallmark of various medical conditions, including type 2 diabetes, obesity, hypertension, metabolic syndrome and polycystic ovary disease $[1,2]$. It may be genetically predisposed in offspring of insulinresistant individuals [3], making insulin resistance a rather severe clinical problem. Resistance to the metabolic actions of insulin is widespread in skeletal, hepatic, adipose and cardiac tissues, and may interfere with glucose transport, hexokinase activity, gene expression, glycogen synthesis and glucose oxidation $[4,5]$. Individuals with insulin resistance exhibit a high incidence of cardiovascular diseases $[1,2]$. Compromised heart function associated with insulin resistance is characterised by impaired cardiac efficiency, reduced ventricular function and coronary heart disease $[1$, 6-9]. These dysfunctions are reminiscent of those found in type 2 diabetic or obese patients with compromised ventric- 
ular function, including prolonged action potential, reduced ventricular wall compliance, diminished cytosolic $\mathrm{Ca}^{2+}$ clearance and prolonged diastolic duration [10-12]. Several potential causes have been postulated for the onset of myopathic changes associated with type 2 diabetes or obesity, and these include oxidative stress, altered glucose metabolism, and impaired function of sarco(endo)plasmic reticulum $\mathrm{Ca}^{2+}$-ATPase and $\mathrm{Na}^{+} / \mathrm{Ca}^{2+}$ exchange $[9,11,13]$. However, it has not been fully established whether prediabetic insulin resistance directly predisposes hearts to cardiac contractile dysfunction in type 2 diabetes or obesity.

Knowledge of insulin signalling has grown dramatically over the past decade. Binding of insulin to its receptor activates tyrosine kinase in the insulin receptor $\beta$ subunit and turns on either IRS-1 and IRS-2, or the Grb2/Sos/Ras/ ERK1/ERK2 pathway [5]. IRS-1/IRS-2-mediated activation of phosphatidylinositol-3 kinase (PI-k3) is believed to be responsible for the metabolic effects of insulin [14], whereas the Grb2/Sos/Ras/ERK pathway barely contributes to insulin-stimulated glucose transport or glycogen synthesis [15]. Several post-receptor components, including the serine/threonine protein kinase Akt, protein tyrosine phosphatase 1B (PTP1B), peroxisome proliferator-activated receptor $\gamma(\mathrm{PPAR} \gamma)$ and mammalian target of rapamycin, have been shown to participate in peripheral glucose metabolism [16-19]. Both dampened and hyperactivated insulin signalling lead to systemic insulin resistance, which may eventually progress to type 2 diabetes and other metabolic disorders $[2,20]$. PTP1B is a negative regulator of insulin signalling, whereas PPAR $\gamma$ agonists have emerged as potent insulin sensitisers $[17,19]$. Both under- and overactivation of cardiac survival factor Akt have been demonstrated in type 2 diabetes or insulin resistance [21-23]. This study was designed to investigate the impact of the antioxidant metallothionein (MT) on oxidative stress induced by prediabetic insulin resistance, and on the stress-induced transcription factor c-Jun, cardiac dysfunction and insulin signalling at the levels of insulin receptor, Akt, PTP1B and PPAR $\gamma$.

\section{Materials and methods}

Experimental animals and induction of whole-body insulin resistance

The experimental procedure was approved by our Institutional Animal Use and Care Committee at the University of North Dakota (Grand Forks, ND, USA) and University of Wyoming (Laramie, WY, USA). All animal procedures were in accordance with NIH animal care standards. In brief, 4-month-old male FVB and MT transgenic mice weighing $\sim 20 \mathrm{~g}$ were randomly divided into two groups each and fed either a cornstarch (providing 68\% of total energy)- or sucrose (providing $68 \%$ of total energy)-based diet formulated by Research Diets (New Brunswick, NJ, USA) for 12 weeks. This sucrose-induced prediabetic model is characterised by hyperinsulinaemic and hypertriglyceridae- mic whole-body insulin resistance $[6,7]$. The mice were maintained in a 12-h/12-h light/dark cycle with free access to tap water. The fur color was used as a marker for MT (dark brown) or wild-type FVB (white) mouse identification as described [24]. For the insulin stimulation study, mice from each group were injected intraperitoneally with insulin (15 $\mathrm{mU} / \mathrm{g}$ body weight) for $10 \mathrm{~min}$ before being killed and the tissue collected.

Intraperitoneal glucose tolerance test, plasma insulin and triglycerides

The i.p. glucose tolerance test was performed as described [25]. After the 12 weeks of dietary feeding, both starch- and sucrose-fed mice were fasted for $12 \mathrm{~h}$ and then given an i.p. injection of glucose $(2 \mathrm{~g} / \mathrm{kg}$ body weight). Blood samples were drawn from the tail $15 \mathrm{~min}$ and immediately before the glucose challenge, as well as 15,60 , and 120 min thereafter. Serum glucose levels were determined using a glucose analyser (Accu-Chek III, model 792; Boehringer Mannheim Diagnostics, Indianapolis, IN, USA). Plasma insulin and triglyceride levels were measured by radioimmunoassay (Linco Research, St Charles, MO, USA).

\section{Isolation of mouse ventricular myocytes}

Hearts were rapidly removed from anaesthetised mice and immediately mounted on a temperature-controlled $\left(37^{\circ} \mathrm{C}\right)$ Langendorff perfusion system. After perfusion with modified Tyrode solution $\left(\mathrm{Ca}^{2+}\right.$ free) for $2 \mathrm{~min}$, the heart was digested for $10 \mathrm{~min}$ with $0.9 \mathrm{mg} / \mathrm{ml}$ collagenase $\mathrm{D}$ (Boehringer Mannheim Biochemicals) in modified Tyrode solution. The modified Tyrode solution ( $\mathrm{pH}$ 7.4) contained the following (in mmol/l): $\mathrm{NaCl} 135, \mathrm{KCl} 4.0, \mathrm{MgCl}_{2} 1.0$, HEPES 10, $\mathrm{NaH}_{2} \mathrm{PO}_{4}$ 0.33 , glucose 10 , butanedione monoxime 10 ; it was gassed with $5 \% \mathrm{CO}_{2}-95 \% \mathrm{O}_{2}$. The digested heart was then removed from the cannula and the left ventricle was cut into small pieces in the modified Tyrode solution. These pieces were gently agitated and the pellet of cells was resuspended in modified Tyrode solution and allowed to settle for another $20 \mathrm{~min}$ at room temperature. During this time, extracellular $\mathrm{Ca}^{2+}$ was added incrementally up to a concentration of $1.2 \mathrm{mmol} / \mathrm{l}$. Isolated myocytes were used for experiments within $8 \mathrm{~h}$ of isolation. For the recording of mechanical properties and intracellular $\mathrm{Ca}^{2+}$ transients as described [26] only rod-shaped myocytes with clear edges were selected.

\section{Cell shortening/relengthening measurements}

The mechanical properties of ventricular myocytes were assessed using a SoftEdge MyoCam system (IonOptix, Milton, MA, USA) [26]. In brief, cells were placed in a Warner chamber mounted on the stage of an inverted microscope (Olympus IX-70; Olympus Optical, Tokyo, 
Japan) and superfused $\left(\sim 1 \mathrm{ml} / \mathrm{min}\right.$ at $\left.25^{\circ} \mathrm{C}\right)$ with a buffer containing (in mmol/1): $\mathrm{NaCl} 131, \mathrm{KCl} 4, \mathrm{CaCl}_{2} 1, \mathrm{MgCl}_{2} 1$, glucose 10, HEPES 10, at $\mathrm{pH} 7.4$. The cells were fieldstimulated with suprathreshold voltage at a frequency of 0.5 $\mathrm{Hz}$ and duration of $3 \mathrm{~ms}$, using a pair of platinum wires placed on opposite sides of the chamber connected to a stimulator (FHC, Bowdoinham, ME, USA). The myocyte being studied was displayed on the computer monitor using an IonOptix MyoCam camera. Changes in cell length during shortening and relengthening were captured with SoftEdge software (IonOptix). Cell shortening and relengthening were assessed according to the following indices: (1) peak shortening (PS), indicative of peak ventricular contractility; (2) time to $90 \%$ PS (TPS 90 ), indicative of systolic duration; (3) time to $90 \%$ relengthening $\left(\mathrm{TR}_{90}\right)$, indicative of diastolic duration; (4) half-width duration, which reflects the duration from $50 \%$ shortening to $50 \%$ relengthening; (5) maximal velocities of shortening $(+\mathrm{d} L / \mathrm{d} t)$ and relengthening $(-\mathrm{d} L /$ $\mathrm{d} t)$, which are indicative of maximal velocities of the rise/fall in ventricular pressure.

\section{Measurement of intracellular $\mathrm{Ca}^{2+}$ transient}

A separate cohort of murine myocytes was loaded with fura2/AM $(0.5 \mu \mathrm{mol} / 1)$ for $10 \mathrm{~min}$ and fluorescence measurements were recorded using a dual-excitation fluorescence photomultiplier system (Ionoptix). Myocytes were placed on an Olympus IX-70 inverted microscope and imaged through a Fluor $\times 40$ oil objective. Cells were exposed to light emitted by a $75 \mathrm{~W}$ lamp and passed through either a 360 or a $380 \mathrm{~nm}$ filter (bandwidths: $\pm 15 \mathrm{~nm}$ ), while being stimulated to contract at $0.5 \mathrm{~Hz}$. Fluorescence emissions were detected at $480-520 \mathrm{~nm}$ by a photomultiplier tube, after first illuminating the cells at $360 \mathrm{~nm}$ for $0.5 \mathrm{~s}$ then at $380 \mathrm{~nm}$ for the duration of the recording protocol ( $333 \mathrm{~Hz}$ sampling rate). The $360 \mathrm{~nm}$ excitation scan was repeated at the end of the protocol and qualitative changes in the intracellular concentration of $\mathrm{Ca}^{2+}\left(\left[\mathrm{Ca}^{2+}\right]\right)_{i}$ were inferred from the ratio of the fura-2 fluorescence intensity (FFI) at the two wavelengths. Fluorescence decay time was also measured as an indication of the intracellular $\mathrm{Ca}^{2+}$ clearing rate [26].
Glutathione and glutathione disulfide assay

Glutathione levels were determined in heart, liver and gastrocnemius and the ratio of glutathione (GSH) : oxidised glutathione (GSSG) was used as an indicator of oxidative stress. In brief, samples were homogenised in 4 volumes $(\mathrm{w} / \mathrm{v})$ of $1 \%$ picric acid. Acid homogenates were centrifuged at 16,000 $\mathrm{g}(30 \mathrm{~min})$ and supernatant fractions collected. Supernatant fractions were assayed for total GSH and GSSG by the standard recycling method. The procedure consisted of using one-half of each sample for GSSG determination and the other half for GSH. Samples for GSSG determination were incubated at room temperature with $2 \mu \mathrm{l}$ of 4-vinyl pyridine per $100 \mu \mathrm{l}$ sample for $1 \mathrm{~h}$ after vigorous vortexing. Incubation with 4 -vinyl pyridine conjugates any GSH present in the sample, so that only GSSG is recycled to GSH without interference by GSH. The GSSG (as GSH $\times 2$ ) was then subtracted from total GSH to determine the actual GSH level and the GSH : GSSG ratio [27].

Western blot analysis of Akt, pAkt, insulin receptor $\beta$, insulin receptor tyrosine phosphorylation, transcription factor c-Jun phosphorylation, PPAR $\gamma$ and PTP1B

The total protein was prepared as described previously [26]. In brief, tissue samples from the ventricles were removed and homogenised in a lysis buffer containing $20 \mathrm{mmol} / \mathrm{l}$ Tris (pH 7.4), $150 \mathrm{mmol} / \mathrm{l} \mathrm{NaCl}, 1 \mathrm{mmol} / \mathrm{l}$ EDTA, $1 \mathrm{mmol} / \mathrm{l}$ EGTA, $1 \%$ Triton, $0.1 \%$ SDS and $1 \%$ protease inhibitor cocktail. Samples were then sonicated for $15 \mathrm{~s}$ and centrifuged at $12,000 \mathrm{~g}$ for $20 \mathrm{~min}$ at $4^{\circ} \mathrm{C}$. The protein concentration of the supernatant was evaluated using Protein Assay Reagent (Bio-Rad, Hercules, CA, USA). Equal amounts $(50 \mu \mathrm{g}$ protein/lane) of the protein from the tissue extraction, or prestained molecular weight markers (GibcoBRL, Gaithersburg, MD, USA) were separated on $10 \%$ or $15 \%$ SDS-polyacrylamide gels in a minigel apparatus (MiniPROTEAN II; Bio-Rad). They were then were transferred electrophoretically to nitrocellulose membranes $(0.2 \mu \mathrm{m}$ pore size; Bio-Rad). Membranes were incubated for $1 \mathrm{~h}$ in a
Table 1 General features of starch- and sucrose-fed FVB and MT mice

Values are means \pm SEM BW body weight; HW heart weight; LW liver weight; $\mathrm{KW}$ kidney weight ${ }^{*} p<0.05$ vs FVB-starch group; $n=20-26$ mice per group $(n=5-6$ for GSH : GSSG assay)

\begin{tabular}{lcccc}
\hline & FVB-starch & FVB-sucrose & MT-starch & MT-sucrose \\
\hline Body weight (g) & $25.2 \pm 0.5$ & $25.4 \pm 0.7$ & $24.4 \pm 0.5$ & $27.1 \pm 0.7$ \\
Heart weight (mg) & $142 \pm 7$ & $139 \pm 4$ & $137 \pm 6$ & $141 \pm 4$ \\
HW:BW (mg/g) & $5.60 \pm 0.21$ & $5.49 \pm 0.17$ & $5.63 \pm 0.23$ & $5.26 \pm 0.14$ \\
Liver weight (g) & $1.27 \pm 0.05$ & $1.32 \pm 0.06$ & $1.19 \pm 0.04$ & $1.30 \pm 0.05$ \\
LW:BW (mg/g) & $50.73 \pm 1.85$ & $52.09 \pm 2.23$ & $48.68 \pm 1.13$ & $48.24 \pm 1.87$ \\
Kidney weight (g) & $0.38 \pm 0.02$ & $0.39 \pm 0.02$ & $0.37 \pm 0.01$ & $0.40 \pm 0.01$ \\
KW:BW (mg/g) & $14.98 \pm 0.57$ & $15.28 \pm 0.59$ & $15.07 \pm 0.40$ & $14.77 \pm 0.39$ \\
Insulin (pmol/l) & $0.100 \pm 0.011$ & $0.556 \pm 0.051^{*}$ & $0.115 \pm 0.011$ & $0.490 \pm 0.058^{*}$ \\
Triglycerides (mmol/l) & $0.78 \pm 0.10$ & $1.68 \pm 0.15^{*}$ & $0.81 \pm 0.10$ & $1.55 \pm 0.17 *$ \\
Heart GSH:GSSG & $1.24 \pm 0.02$ & $1.14 \pm 0.03^{*}$ & $1.23 \pm 0.02$ & $1.19 \pm 0.02$ \\
Liver GSH:GSSH & $1.49 \pm 0.04$ & $1.29 \pm 0.03^{*}$ & $1.43 \pm 0.05$ & $1.30 \pm 0.04^{*}$ \\
Gastrocnemius GSH:GSSG & $1.40 \pm 0.08$ & $1.20 \pm 0.06^{*}$ & $1.47 \pm 0.07$ & $1.30 \pm 0.04^{*}$ \\
\hline
\end{tabular}




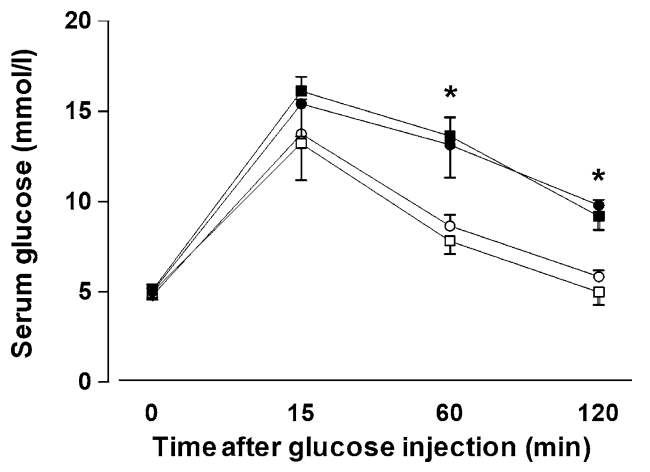

Fig. 1 I.p. glucose tolerance test. Serum glucose concentrations in response to an i.p. glucose challenge ( 2 g glucose $/ \mathrm{kg}$ body weight) in starch- and sucrose-fed FVB and MT mice are shown. The mice were fasted for $12 \mathrm{~h}$ before tests were conducted. Open squares, FVB starch-fed; filled squares, FVB sucrose-fed; open circles, MT starchfed; filled circles, MT sucrose-fed. Results are means \pm SEM; $n=9-10$ mice per group; ${ }^{*} p<0.05$ for difference between the starch- and sucrose-fed groups

Fig. 2 Contractile properties of left ventricular myocytes from starch- and sucrose-fed FVB and MT mouse hearts. a Resting cell length; b peak shortening (normalised to cell length); c maximal velocity of shortening $(+\mathrm{d} L / \mathrm{d} t)$ and relengthening $(-\mathrm{d} L / \mathrm{d} t)$; d halfwidth duration; e TPS $_{90}$; f $\mathrm{TR}_{90}$. Results are means \pm SEM; $n=50$ cells/group; $* p<0.05$ vs FVB-starch group; $\# p<0.05$ vs FVB-sucrose group a

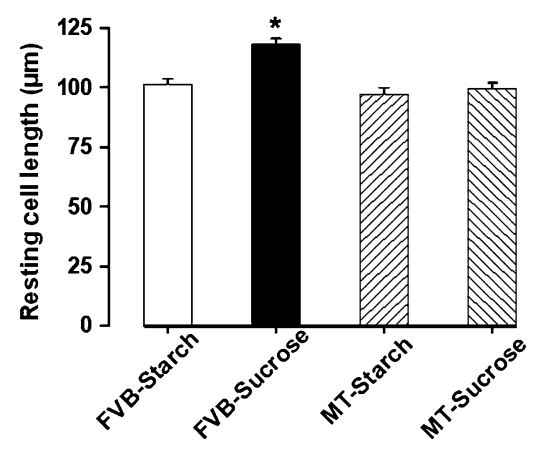

C

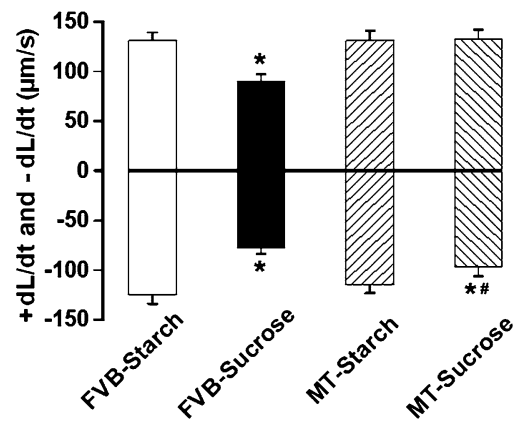

e

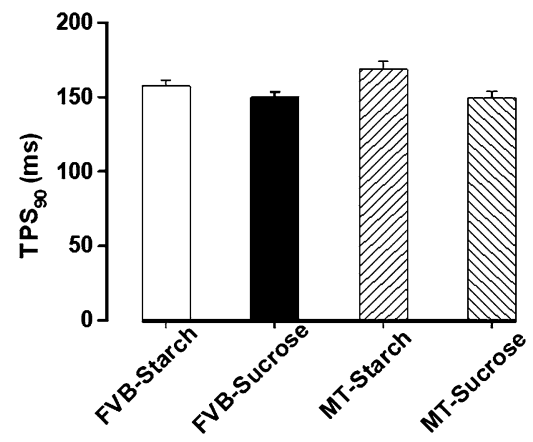

b

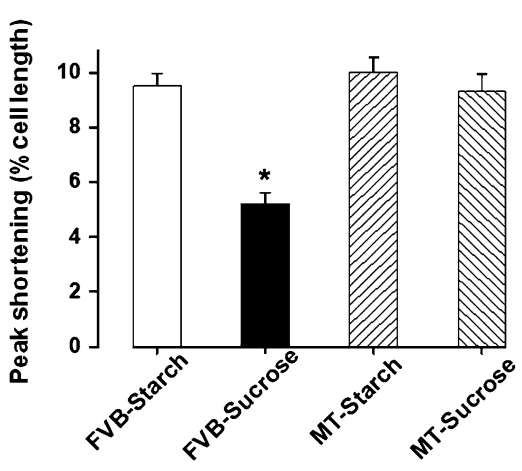

d

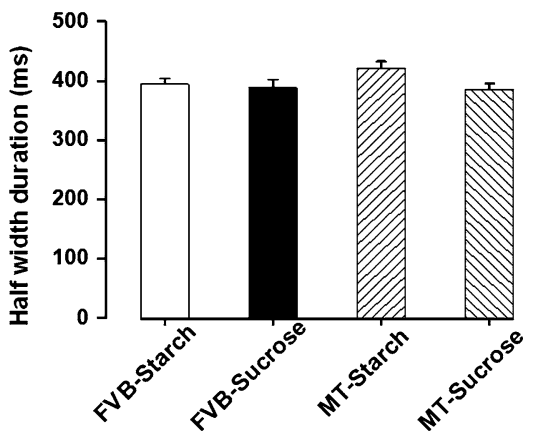

f

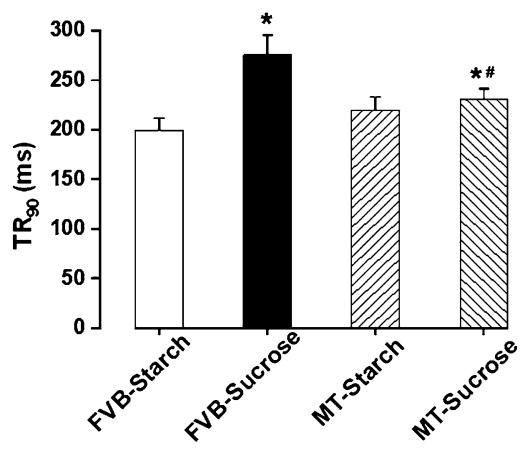


Data analysis

Data are expressed as means \pm SE. Statistical comparisons were performed by ANOVA, followed by Newman-Keuls post hoc test. Significance was defined as $p<0.05$.

\section{Results}

Experimental animals

There was no difference in body, heart, liver and kidney weight or organ : body weight ratio among FVB-starch-fed, FVB-sucrose-fed, MT-starch-fed and MT-sucrose-fed mice. Plasma levels of insulin and triglycerides were significantly higher in sucrose-fed mice than in starch-fed mice (Table 1). Following the acute i.p. glucose challenge $(2 \mathrm{~g} / \mathrm{kg}$ body weight), plasma glucose levels in the starch-fed FVB or MT mice peaked at $15 \mathrm{~min}$, then started to decline, nearly reaching the baseline value by $120 \mathrm{~min}$. However, in the sucrose-fed FVB or MT mice, post-challenge glucose levels remained at much higher levels between 15 and $120 \mathrm{~min}$ (Fig. 1), indicating that these mice had impaired glucose clearance, which is indicative of glucose intolerance and insulin resistance. There was no difference in basal fasting glucose levels among the four mouse groups, excluding the presence of diabetes in sucrose-fed mice following the 12week sucrose feeding regimen. The GSH : GSSG ratio was significantly reduced in heart, liver and gastrocnemius muscle from sucrose-fed FVB mice (Table 1), a sign of enhanced oxidative stress following dietary sucrose feeding. Interestingly, cardiac overexpression of metallothionein restored the GSH/GSSG levels in heart, but not in liver or gastrocnemius muscle of the sucrose-fed mice.

Mechanical and fluorescent properties of myocytes from starch- and sucrose-fed mice

Insulin resistance is known to increase the propensity to cardiac hypertrophy. In sucrose-fed FVB mice we observed a significantly longer resting cell length than in starch-fed FVB mice, but this was abolished by cardiac overexpression of metallothionein. Both PS amplitude and $+\mathrm{d} L / \mathrm{d} t /-\mathrm{d} L / \mathrm{d} t$ were significantly depressed in myocytes from the sucrosefed FVB group, compared with those from starch-fed FVB mice. Myocytes from sucrose-fed FVB mice displayed normal $\mathrm{TPS}_{90}$ and a significantly prolonged $\mathrm{TR}_{90}$ compared with those from starch-fed FVB mice. In all four mouse groups, there was no difference in half-width duration, an index of the duration of late-phase contraction and earlyphase relaxation. Interestingly, metallothionein effectively protected ventricular myocytes from the mechanical dysfunctions induced by the sucrose diet (Fig. 2). Our further experiments using intracellular fura-2 fluorescence revealed that the electrically stimulated rise in FFI $(\Delta \mathrm{FFI})$ was depressed and the intracellular $\mathrm{Ca}^{2+}$ clearing rate slower in myocytes from sucrose-fed FVB myocytes. This is consistent with the reduced PS and prolonged $\mathrm{TR}_{90}$ found in sucrose myocytes. Similar to its effect on cell shortening, metallothionein abolished the sucrose-induced decrease in $\triangle$ FFI and prolongation of intracellular $\mathrm{Ca}^{2+}$ clearing. Resting intracellular $\mathrm{Ca}^{2+}$ levels (resting FFI) were unaltered by diet or metallothionein (Fig. 3).

Changes of protein expression of Akt, pAkt, insulin receptor $\beta$, insulin receptor tyrosine phosphorylation, PPAR $\gamma$, PTP1B and phosphorylation of transcription factor c-Jun

Insulin resistance is associated with the interruption of insulin signalling at the insulin receptor and post-receptor levels [2]. To causally relate changes of cardiomyocyte
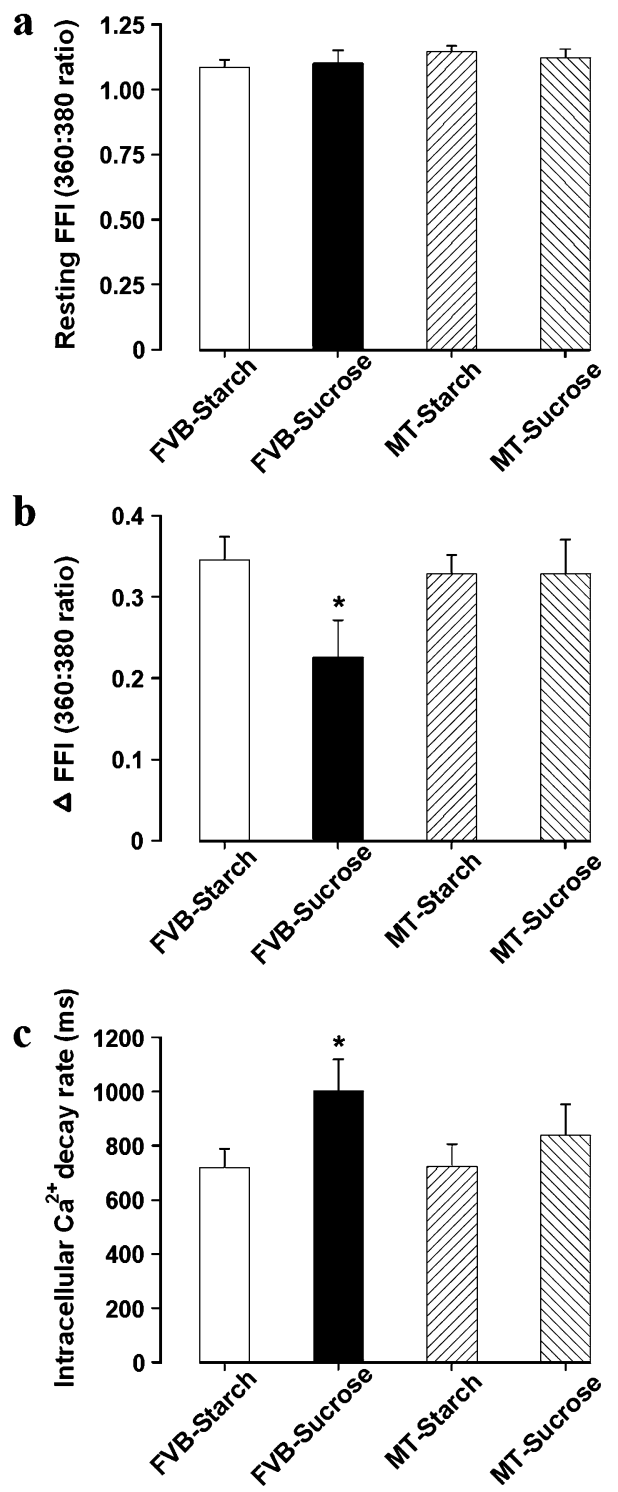

Fig. 3 Intracellular $\mathrm{Ca}^{2+}$ transient properties in ventricular myocytes from starch- and sucrose-fed FVB and MT mouse hearts. a Resting intracellular $\mathrm{Ca}^{2+}$ FFI; $\mathbf{b}$ electrically stimulated increase in FFI $(\triangle \mathrm{FFI})$; $\mathbf{c}$ intracellular $\mathrm{Ca}^{2+}$ transient decay rate. Values are means \pm SEM; $n=15-16$ cells/group; * $p<0.05$ vs FVB-starch group 
Fig. 4 a Representative western blot exhibiting total and phosphorylated Akt levels in ventricles from starch (ST)- and sucrose (SU)-fed FVB and MT mice challenged with or without insulin $(15 \mathrm{mU} / \mathrm{g}$, i.p. for 10 min). Anti-Akt and anti-phospho-Akt (pAkt) antibodies were used. b Total Akt expression; c pAkt expression; $\mathbf{d}$ pAkt : Akt ratio. Values are means \pm SEM; $n=10-13$ for non-insulin treatment; $n=5-6$ for insulin injection; $* p<0.05$ vs FVB-starch-fed; $\# p<0.05$ vs corresponding noninsulin-stimulated group a

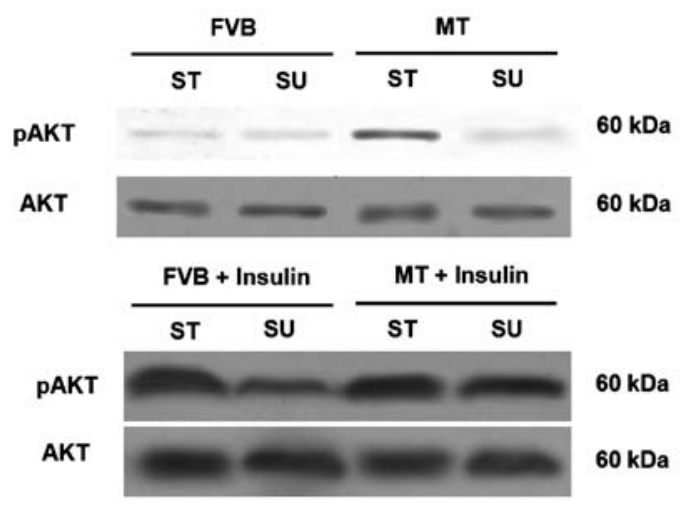

c

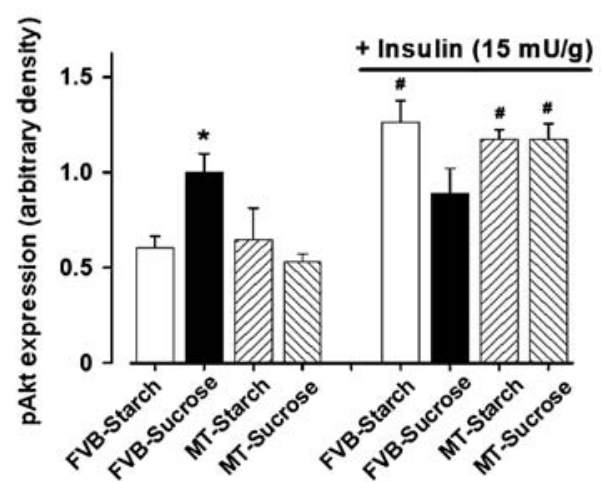

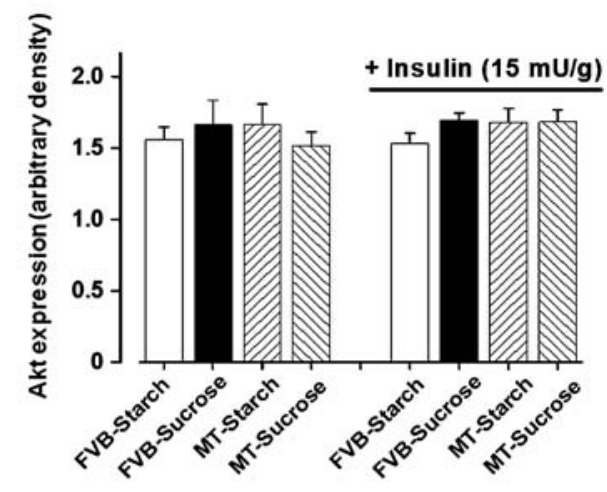

d

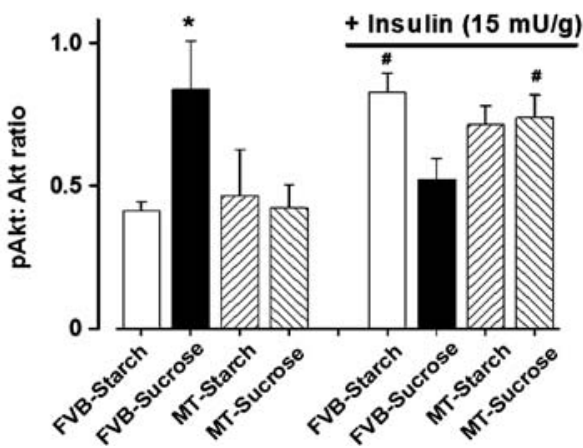

mechanical function to levels of insulin signalling, we examined the effect of insulin resistance and the antioxidant metallothionein on cardiac expression of Akt, pAkt, insulin receptor $\beta$, insulin receptor tyrosine phosphorylation, PPAR $\gamma$ and PTP1B. Our results indicated that insulin resistance induced by sucrose feeding did not affect total
Akt expression in the hearts, either in the basal or in the insulin-stimulated state. However, the sucrose diet significantly enhanced phosphorylation of Akt (both pAkt and the pAkt : Akt ratio) in the absence of insulin stimulation. Acute insulin stimulation $(15 \mathrm{mU} / \mathrm{g}, 10 \mathrm{~min})$ enhanced Akt phosphorylation in starch-fed FVB mice but not in sucrose
Fig. 5 a, b Representative western blots exhibiting insulin receptor expression and insulin receptor tyrosine phosphorylation (Tyr1146) in ventricles from starch (ST)- and sucrose (SU)-fed FVB and MT mice challenged without (a) or with (b) insulin $(15 \mathrm{mU} / \mathrm{g}$, i.p. for 10 min). Antibodies used: anti-insulin receptor and anti-phosphoinsulin receptor (Tyr1146).

c Insulin receptor expression; d anti-phospho-insulin receptor (Tyr1146) expression. Values are means \pm SEM; $n=6-12$; $\dagger \dagger p<0.05$ vs all other groups with the same insulin treatment; ${ }^{*} p<0.05$ vs corresponding noninsulin stimulated groups; $\# p<0.05$ vs insulin-stimulated FVB-starch group a
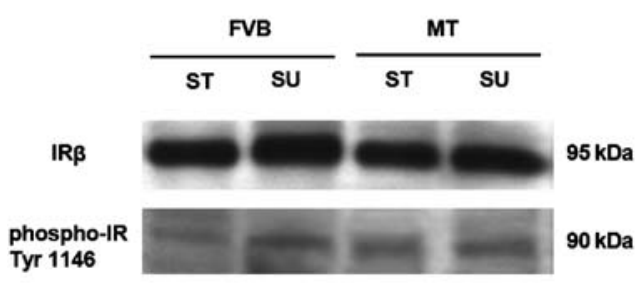

c

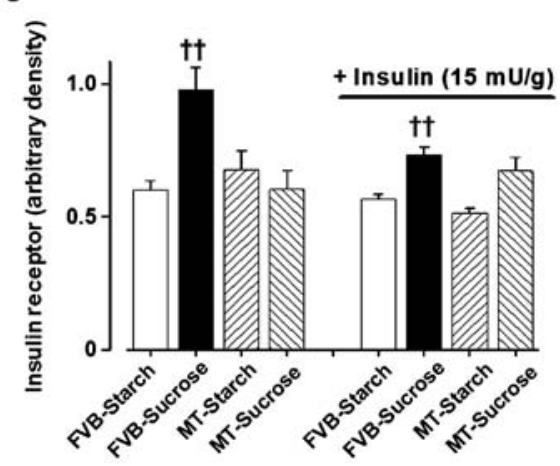

b
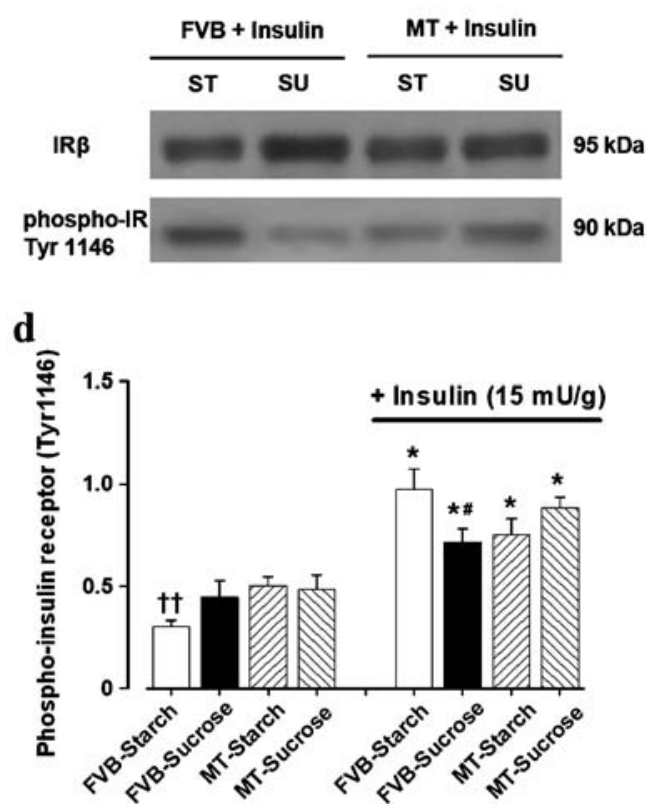
Fig. 6 a A representative western blot exhibiting PPAR $\gamma$, PTP1B and phosphorylated c-Jun expression in ventricles from starch (ST)- and sucrose (SU)-fed FVB and MT mouse hearts in the absence of insulin injection. Antibodies used: antiPPAR $\gamma$, anti-PTP1B and antiphospho-c-Jun(Ser63)II. b PPAR $\gamma$ expression;

(c) PTP1B expression;

(d) phosphorylation of c-Jun

Values are means \pm SEM; $n=6-8$; $* p<0.05$ vs FVB-starch group; $\# p<0.05$ vs FVB-sucrose group a

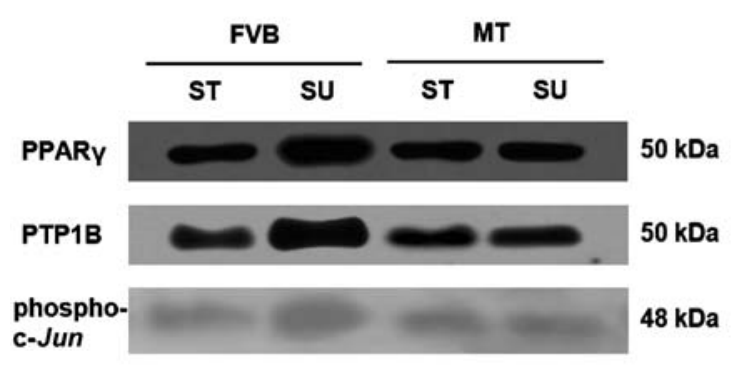

phospho-
C-Jun

$8 \mathrm{kDa}$

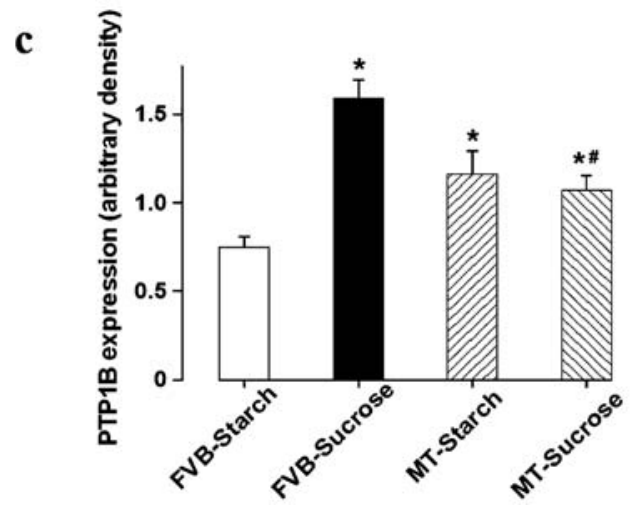

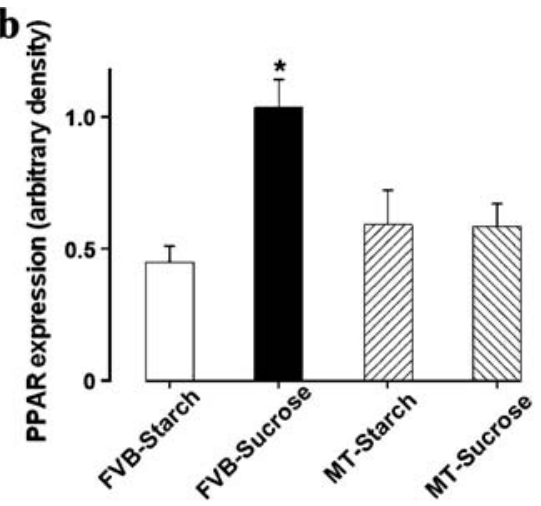

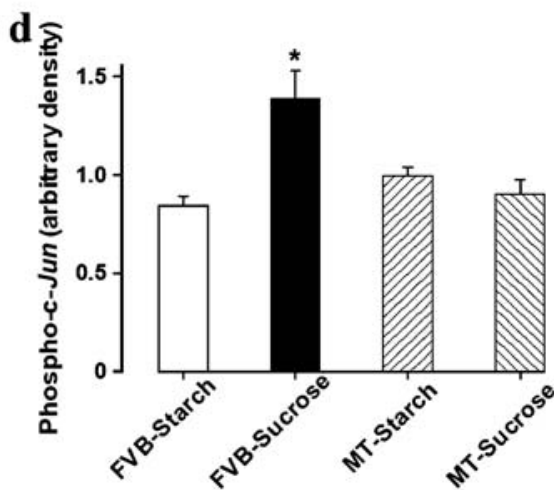

FVB group. Interestingly, metallothionein ablated insulinresistance-induced basal or tonic Akt hyper-phosphorylation and insulin-stimulated Akt hypo-phosphorylation without affecting total Akt expression (Fig. 4). Further study revealed that insulin resistance significantly enhanced expression of insulin receptor $\beta$ (basal and insulin-stimulated) and basal insulin receptor tyrosine phosphorylation. Although insulin stimulation significantly enhanced tyrosine phosphorylation of insulin receptors in both FVB groups, the fold increase of insulin receptor tyrosine phosphorylation was much lower in the sucrose-fed group (0.43-fold) than in the starch-fed group (2.20-fold). Metallothionein corrected the upregulation of insulin receptor $\beta$ that was induced by insulin resistance, and partially restored insulin-stimulated insulin receptor tyrosine phosphorylation in sucrose-fed mice. The metallothionein transgene itself enhanced basal tyrosine phosphorylation of the insulin receptor (Fig. 5). Our results for sucrose-fed mouse hearts also indicated significantly enhanced expression both of the key post-insulin receptor signalling molecules PPAR $\gamma$ and PTP1B, and of the phosphorylated transcription factor c-Jun. This may be blunted or attenuated by metallothionein transgene. In the starch-fed group, metallothionein itself did not affect expression of PPAR $\gamma$ and phosphorylated c-Jun, but did significantly enhance expression of PTP1B (Fig. 6).

\section{Discussion}

The major findings of our study are that prediabetic insulin resistance elicits glucose intolerance, hyperinsulinaemia, hypertriglyceridaemia, oxidative stress, cardiac contractile dysfunction and impaired intracellular $\mathrm{Ca}^{2+}$ handling. The cardiac contractile and intracellular $\mathrm{Ca}^{2+}$ dysfunctions that were induced by insulin resistance were causally associated with enhanced tonic phosphorylation of Akt, blunted insulin-stimulated phosphorylation of Akt, increased expression of insulin receptor $\beta$ and insulin receptor tyrosine phosphorylation, reduced response of insulin-stimulated insulin receptor tyrosine phosphorylation, and elevated expression of PPAR $\gamma$, PTP1B, and the transcription factor c-Jun. Interestingly, these alterations in oxidative status, contractile function and insulin signalling that were induced by insulin resistance were significantly attenuated or abolished by cardiac overexpression of the antioxidant metallothionein. Since the metallothionein transgene did not affect whole-body glucose intolerance, hyperinsulinaemia and hypertriglyceridaemia, our data suggest that cardiac contractile dysfunction and altered insulin signalling as induced by insulin resistance are probably associated with oxidative stress in the mouse hearts. This is supported by our finding that metallothionein alleviated enhanced oxidative stress induced by the sucrose diet, but only in the heart, and not in liver or skeletal muscle.

Insulin resistance is associated with type 2 diabetes, obesity and dyslipidaemia [1,2], and is thought to impair cardiac contractile function through increased blood pressure via sympathetic activation, as well as through stimulation of the renin-angiotensin system, and direct alteration of cardiac myocyte function $[1,6,7,25]$. The alleviation of insulin resistance by pharmacological intervention and lifestyle modification, including diet, weight loss and physical exercise, improves cardiac function and reduces blood pressure $[2,4]$. Angiotensin-converting enzyme inhibitors, vasodila- 
tory $\beta$-blockers and L-type $\mathrm{Ca}^{2+}$ channel blockers have been reported to improve insulin sensitivity and beneficially affect insulin resistance [28]. Our 12-week starch/sucrose dietary feeding protocol elicited hyperinsulinaemia, hypertriglyceridaemia and glucose intolerance without changes in body weight, organ weight and fasting blood glucose between FVB and MT mice, ruling out the possibility that obesity and diabetes were present in and contributed to results for our insulin-resistant model. Data from our study reveal that ventricular myocytes from sucrose-fed mice exhibited depressed PS amplitude, reduced $+\mathrm{d} L / \mathrm{d} t /-\mathrm{d} L / \mathrm{d} t$, and prolonged duration of relaxation $\left(\mathrm{TR}_{90}\right)$ associated with normal duration of contraction $\left(\mathrm{TPS}_{90}\right)$ and half-width duration of contraction and relaxation.

Most of these data are consistent with our previous findings using a similar insulin resistance model in rats [7, $25]$. The fact that our early observation showed normal $\mathrm{TR}_{90}$ and $+\mathrm{d} L / \mathrm{d} t /-\mathrm{d} L / \mathrm{d} t[7]$ or shortened TPS ${ }_{90}$ [25] after sucrose diet intake may result from the different feeding duration (78 weeks or 10 weeks) $[7,25]$ and difference in species. These mechanical defects are quite similar to those reported in ventricular myocytes from full-blown diabetes, all of which are characteristic of diabetic cardiomyopathy $[12,13$, 26]. The impaired intracellular $\mathrm{Ca}^{2+}$ handling, observed in the form of a reduced intracellular $\mathrm{Ca}^{2+}$ clearance rate and a reduced intracellular $\mathrm{Ca}^{2+}$ rise $(\Delta \mathrm{FFI})$ in sucrose-fed FVB mouse myocytes, is consistent with and most probably directly responsible for the prolonged relaxation $\left(\mathrm{TR}_{90}\right)$ and reduced peak shortening in these cells. The normal TPS $S_{90}$ and half-width durations seem to indicate that prolonged relaxation may occur during the late phase of relaxation rather than in early ones. In addition, our data revealed prolonged relaxation duration $\left(\mathrm{TR}_{90}\right)$ but normal maximal velocity of relaxation $(-\mathrm{d} L / \mathrm{d} t)$. This discrepancy may indicate that protein(s) responsible for rapid relaxation (i.e. the rapid phase of ventricular filling during the diastole), e.g. myosin heavy-chain isozyme, may be normal, whereas cellular machineries responsible for reduced-phase ventricular filling or slow cytosolic $\mathrm{Ca}^{2+}$ extrusion (e.g. $\mathrm{Na}^{+} / \mathrm{Ca}^{2+}$ exchange, mitochondrial or sarcolemmal $\mathrm{Ca}^{2+}$ pumps) are defective. Further study is warranted to elucidate the expression and function of these myocardial proteins.

It appears that the effects of type 2 diabetes on the heart are more subtle than those of type 1 diabetes and may become visible only after metabolic challenge such as increased work load $[29,30]$. The existence of cardiomyocyte dysfunction has been shown in some $[6,7,25,31-34]$ but not all [35] animal models of type 2 diabetes or insulin resistance. The mechanical and intracellular $\mathrm{Ca}^{2+}$ defects triggered by insulin resistance may be alleviated by metallothionein, in a manner similar to its effect on diabetes-induced cardiac contractile dysfunctions [13]. Metallothionein protection of cardiomyocyte function in type 1 diabetes was due to its antioxidant action $[13,36]$. It is likely that reduction of oxidative stress was also responsible for the protective effect of metallothionein on cardiac contractile dysfunction associated with insulin resistance, a notion supported by its antagonism against the sucrose-diet-induced reduction of GSH : GSSG ratio and against hyper- phosphorylation of the transcription factor c-Jun, a key signalling molecule stimulated by enhanced oxidative stress [37]. C-Jun is a unique transcription factor controlling cell survival through regulation of cell cycle regulators such as p53, p21 and p16 [37].

More recently, a unique concept of the antioxidant protective mechanism of metallothionein against diabetic cardiomyopathy has been postulated. Metallothionein was shown to alleviate enhanced superoxide generation, 3nitrotyrosine formation and nitrosative damage in streptozotocin-induced diabetes [38]. Hyperglycaemia and hyperinsulinaemia contribute to the accumulation of reactive oxygen species, reactive nitrogen species and oxidative stress $[13,38,39]$, although the role of c-Jun and other transcription factors such as Fos in oxidative-stress-associated myocardial function has not been elucidated.

Our current study revealed upregulation of several insulin-signalling molecules in insulin-resistant FVB mice, both at the receptor and post-receptor levels. Perhaps the most interesting finding was that alterations of these insulinsignalling molecules (insulin receptor $\beta$, insulin receptor tyrosine phosphorylation, Akt phosphorylation, PTP1B and $\operatorname{PPAR} \gamma)$ are associated with altered contractile function in either insulin-sensitive or insulin-resistant mice, with or without antioxidant protection from metallothionein. Our finding of improved function with antioxidant metallothionein is consistent with an earlier report that the abrogation of oxidative stress may improve insulin sensitivity in the Ren2 rat model of tissue angiotensin II overexpression [40]. Defects of insulin signalling often include reduced expression and/or function of insulin receptor and IRS-1, as well as diminished insulin receptor kinase activation and tyrosine phosphorylation of IRS-1, and defective activation of PI-3K [41-43]. The elevated expression of insulin receptor $\beta$, insulin receptor tyrosine phosphorylation and tonic Akt phosphorylation in insulin-resistant FVB mice may indicate a pseudo-hyperactivity of insulin signalling, possibly due to a feedback upregulatory mechanism as a result of desensitised insulin signalling after 12 weeks of sucrose feeding. What is detrimental for such high tonic phosphorylation of the insulin receptor and Akt is that subsequent insulin stimulation cannot generate any or only an inadequate response - the condition of insulin resistance. This is shown by our finding of blunted or reduced insulinstimulated phosphorylation of insulin receptor tyrosine phosphorylation and Akt. It is perhaps not surprising that sucrose-induced insulin resistance is accompanied by upregulated expression of PTP1B, the negative modulator of insulin signalling. It has been shown that reactive oxygen species is essential for optimal tyrosine phosphorylation and insulin signalling in response to diverse stimuli $[44,45]$. Protein tyrosine phosphatases (PTPs) are extremely sensitive to inhibition by reactive oxygen species via reversible oxidation [45]. Although oxidative stress may facilitate a tyrosine-phosphorylation-dependent cellular signalling response of insulin by transiently inactivating the PTPs that would normally suppress the insulin signal, excessive accumulation of reactive oxygen species under insulin resistance and diabetes has been reported to upregulate the 
redox-sensitive protein PTP1B [46]. On the other hand, the sucrose-induced increase in PPAR $\gamma$, the adipocyte-predominant transcription factor found in low abundance in the heart [47], may serve as an insulin sensitiser to regulate glucose and lipid homeostasis [17]. PPAR $\gamma$ may be upregulated in a compensatory manner by excessive accumulation of reactive oxygen species [48], as seen in our current study. It can be speculated that the antioxidant metallothionein reconciles the imbalanced redox status triggered by insulin resistance under the present experimental setting, thus restoring the normal insulin-signalling mechanisms at the levels of insulin receptor $\beta$, insulin receptor tyrosine phosphorylation, Akt phosphorylation, PTP1B and PPAR $\gamma$. However, further studies using either confirmatory functional (such as pharmacological) interventions or specific gene overexpression or deletion approaches are warranted to validate the involvement of these insulin-signalling molecules.

With regard to the experimental limitations of our study, it should be noted that the absolute heart weights or heart : body weight ratios were not different among the four animal groups, whereas cardiomyocytes from sucrose-fed FVB mice were significantly longer than in all other groups, suggesting some form of cardiac remodelling. Possible speculative explanations of this 'cardiac hypertrophy' include elevated circulating levels of growth factors such as IGF-1. However, our study failed to provide any such evidence with regard to the observed discrepancy between heart weight and myocyte length. In addition, we did not establish any mechanism of action, such as altered mitochondrial function, behind the elevated oxidative stress seen in sucrose-fed mice. Further study is warranted to define the link between insulin resistance and oxidative stress so that optimal therapeutic regimens may be planned to alleviate possible organ damage under insulin resistance and/or oxidative stress.

Oxidative stress and impaired cardiac contractile function have been demonstrated in insulin-resistant states such as type 2 diabetes, hypertension and obesity [49]. A highfructose diet has been shown to reduce mRNA expression of antioxidant catalase [50]. Our present study indicated that insulin resistance contributes to cardiac contractile dysfunctions, oxidative stress and activation of the transcription factor c-Jun, as well as to impaired insulin signalling, which may be alleviated by overexpression of the antioxidant metallothionein in the heart. Although our study sheds some light on the interaction between oxidative stress, insulin signalling and the cardiac defects associated with insulin resistance, the pathogenesis of cardiac contractile dysfunction under insulin resistance still deserves further in-depth investigation. It is important to delineate the insulin-signalling mechanism under oxidative stress and to understand the transition from an insulin-sensitive to an insulin-resistant state. It is also imperative that we understand the direct role of long-lasting insulin resistance on cardiac dynamics in response to oxidative stress.
Acknowledgements This work was supported in part by grants from the American Heart Association Pacific Mountain Affiliate (\#0355521Z) and the American Diabetes Association (7-0-RA-21) to J. Ren.

\section{References}

1. Reaven GM (1995) Pathophysiology of insulin resistance in human disease. Physiol Rev 75:473-486

2. Wang CC, Goalstone ML, Draznin B (2004) Molecular mechanisms of insulin resistance that impact cardiovascular biology. Diabetes 53:2735-2740

3. Vauhkonen I, Niskanen L, Vanninen E, Kainulainen S, Uusitupa M, Laakso M (1998) Defects in insulin secretion and insulin action in non-insulin-dependent diabetes mellitus are inherited. Metabolic studies on offspring of diabetic probands. J Clin Invest 101:86-96

4. Matthaei S, Stumvoll M, Kellerer M, Haring HU (2000) Pathophysiology and pharmacological treatment of insulin resistance. Endocr Rev 21:585-618

5. Cusi K, Maezono K, Osman A et al (2000) Insulin resistance differentially affects the PI 3-kinase- and MAP kinase-mediated signaling in human muscle. J Clin Invest 105:311-320

6. Dutta K, Podolin DA, Davidson MB, Davidoff AJ (2001) Cardiomyocyte dysfunction in sucrose-fed rats is associated with insulin resistance. Diabetes 50:1186-1192

7. Hintz KK, Ren J (2002) Prediabetic insulin resistance is not permissive to the development of cardiac resistance to insulinlike growth factor I in ventricular myocytes. Diabetes Res Clin Pract 55:89-98

8. Davidoff AJ, Mason MM, Davidson MB et al (2004) Sucroseinduced cardiomyocyte dysfunction is both preventable and reversible with clinically relevant treatments. Am J Physiol Endocrinol Metab 286:E718-E724

9. Mazumder PK, O’Neill BT, Roberts MW et al (2004) Impaired cardiac efficiency and increased fatty acid oxidation in insulinresistant $o b / o b$ mouse hearts. Diabetes 53:2366-2374

10. Gargiulo P, Jacobellis G, Vaccari V, Andreani D (1998) Diabetic cardiomyopathy: pathophysiological and clinical aspects. Diabet Nutr Metab 11:336-346

11. Ren J, Ceylan-Isik AF (2004) Diabetic cardiomyopathy: do women differ from men? Endocrine 25:73-83

12. Ren J, Davidoff AJ (1997) Diabetes rapidly induces contractile dysfunctions in isolated ventricular myocytes. Am J Physiol 272:H148-H158

13. Ye G, Metreveli NS, Ren J, Epstein PN (2003) Overexpression of metallothionein reverses diabetes induced functional deficits in diabetic cardiomyocytes by inhibiting ROS production. Diabetes 52:777-783

14. Dorrestijn J, Ouwens DM, Van den Berghe N, Bos JL, Maassen JA (1996) Expression of a dominant-negative Ras mutant does not affect stimulation of glucose uptake and glycogen synthesis by insulin. Diabetologia 39:558-563

15. Lazar DF, Wiese RJ, Brady MJ et al (1995) Mitogen-activated protein kinase kinase inhibition does not block the stimulation of glucose utilisation by insulin. J Biol Chem 270:2080120807

16. Hinault C, Mothe-Satney I, Gautier N, Lawrence JC Jr, Van Obberghen E (2004) Amino acids and leucine allow insulin activation of the PKB/mTOR pathway in normal adipocytes treated with wortmannin and in adipocytes from $\mathrm{db} / \mathrm{db}$ mice. FASEB J 18:1894-1896

17. Rangwala SM, Lazar MA (2004) Peroxisome proliferator-activated receptor gamma in diabetes and metabolism. Trends Pharmacol Sci 25:331-336

18. Um SH, Frigerio F, Watanabe M et al (2004) Absence of S6K1 protects against age- and diet-induced obesity while enhancing insulin sensitivity. Nature 431:200-205 
19. Zabolotny JM, Haj FG, Kim YB et al (2004) Transgenic overexpression of protein-tyrosine phosphatase $1 \mathrm{~B}$ in muscle causes insulin resistance, but overexpression with leukocyte antigen-related phosphatase does not additively impair insulin action. J Biol Chem 279:24844-24851

20. Fisher TL, White MF (2004) Signaling pathways: the benefits of good communication. Curr Biol 14:R1005-R1007

21. Tomas E, Lin YS, Dagher Z et al (2002) Hyperglycemia and insulin resistance: possible mechanisms. Ann $N$ Y Acad Sci 967:43-51

22. Huisamen B (2003) Protein kinase B in the diabetic heart. Mol Cell Biochem 249:31-38

23. Ren J, Duan J, Hintz KK, Ren BH (2003) High glucose induces cardiac insulin-like growth factor I resistance in ventricular myocytes: role of Akt and ERK activation. Cardiovasc Res 57:738-748

24. Kang YJ, Chen Y, Yu A, Voss-McCowan M, Epstein PN (1997) Overexpression of metallothionein in the heart of transgenic mice suppresses doxorubicin cardiotoxicity. J Clin Invest 100:1501-1506

25. Hintz KK, Aberle NS, Ren J (2003) Insulin resistance induces hyperleptinemia, cardiac contractile dysfunction but not cardiac leptin resistance in ventricular myocytes. Int $\mathrm{J}$ Obes Relat Metab Disord 27:1196-1203

26. Duan J, Zhang HY, Adkins SD et al (2003) Impaired cardiac function and IGF-I response in myocytes from calmodulindiabetic mice: role of Akt and RhoA. Am J Physiol Endocrinol Metab 284:E366-E376

27. Ren J, Roughead ZK, Wold LE et al (2003) Increases in insulinlike growth factor-1 level and peroxidative damage after gestational ethanol exposure in rats. Pharmacol Res 47:341-347

28. Imazu M (2002) Hypertension and insulin disorders. Curr Hypertens Rep 4:477-482

29. Rösen P, Herberg L, Reinauer H (1986) Different types of postinsulin receptor defects contribute to insulin resistance in hearts of obese Zucker rats. Endocrinology 119:1285-1291

30. Lopaschuk GD, Russell JC (1991) Myocardial function and energy substrate metabolism in the insulin-resistant JCR:LA corpulent rat. J Appl Physiol 71:1302-1308

31. Schaffer SW, Mozafferi M (1996) Abnormal mechanical function in diabetes: relation to myocardial calcium handling. Coron Artery Dis 7:109-115

32. Schaffer SW, Ballard-Croft C, Boerth S, Allo SN (1997) Mechanisms underlying depressed $\mathrm{Na}^{+} / \mathrm{Ca}^{2+}$ exchanger activity in the diabetic heart. Cardiovasc Res 34:129-136

33. Shimoni Y, Ewart HS, Severson D (1998) Type I and II models of diabetes produce different modifications of $\mathrm{K}^{+}$currents in rat heart: role of insulin. J Physiol 507:485-496

34. Ren J, Sowers JR, Walsh MF, Brown RA (2000) Reduced contractile response to insulin and insulin-like growth factor I in ventricular myocytes from genetically obese Zucker rats. Am J Physiol Heart Circ Physiol 279:H1708-H1714

35. Clark TA, Pierce GN (2000) Cardiovascular complications of non-insulin-dependent diabetes. The JCR:LA-cp rat. J Pharmacol Toxicol Methods 43:1-10

36. Liang Q, Carlson EC, Donthi R, Kralik P, Xia S, Epstein PN (2002) Overexpression of metallothionein reduces diabetic cardiomyopathy. Diabetes 51:174-181
37. Vulin AI, Stanley FM (2004) Oxidative stress activates the plasminogen activator inhibitor type 1 (PAI-1) promoter through an AP-1 response element and cooperates with insulin for additive effects on PAI-1 transcription. J Biol Chem 279: 25172-25178

38. Cai L, Wang J, Li Y et al (2005) Inhibition of superoxide generation and associated nitrosative damage is involved in metallothionein prevention of diabetic cardiomyopathy. Diabetes $54: 1829-1837$

39. Cai L, Kang YJ (2001) Oxidative stress and diabetic cardiomyopathy: a brief review. Cardiovasc Toxicol 1:181-193

40. Blendea MC, Jacobs D, Stump CS et al (2005) Abrogation of oxidative stress improves insulin sensitivity in the Ren2 rat model of tissue angiotensin II overexpression. Am J Physiol Endocrinol Metab 288:E353-E359

41. Goodyear LJ, Giorgino F, Sherman LA, Carey J, Smith RJ, Dohm GL (1995) Insulin receptor phosphorylation, insulin receptor substrate-1 phosphorylation, and phosphatidylinositol 3-kinase activity are decreased in intact skeletal muscle strips from obese subjects. J Clin Invest 95:2195-2204

42. Saad MJ, Araki E, Miralpeix M, Rothenberg PL, White MF, Kahn CR (1992) Regulation of insulin receptor substrate-1 in liver and muscle of animal models of insulin resistance. J Clin Invest 90:1839-1849

43. Heydrick SJ, Jullien D, Gautier N et al (1993) Defect in skeletal muscle phosphatidylinositol 3-kinase in obese insulin-resistant mice. J Clin Invest 91:1358-1366

44. McClung JP, Roneker CA, Mu W et al (2004) Development of insulin resistance and obesity in mice overexpressing cellular glutathione peroxidase. Proc Natl Acad Sci U S A 101:88528857

45. Meng TC, Buckley DA, Galic S, Tiganis T, Tonks NK (2004) Regulation of insulin signaling through reversible oxidation of the protein-tyrosine phosphatases TC45 and PTP1B. J Biol Chem 279:37716-37725

46. Hirata AE, Alvarez-Rojas F, Carvalheira JB, Carvalho CR, Dolnikoff MS, Abdalla Saad MJ (2003) Modulation of IR/ PTP1B interaction and downstream signaling in insulin sensitive tissues of MSG-rats. Life Sci 73:1369-1381

47. Gilde AJ, van der Lee KA, Willemsen PH et al (2003) Peroxisome proliferator-activated receptor (PPAR) alpha and PPARbeta/delta, but not PPARgamma, modulate the expression of genes involved in cardiac lipid metabolism. Circ Res 92: $518-524$

48. Iwashima Y, Eto M, Horiuchi S, Sano H (1999) Advanced glycation end product-induced peroxisome proliferator-activated receptor gamma gene expression in the cultured mesangial cells. Biochem Biophys Res Commun 264:441-448

49. Ceddia RB, Koistinen HA, Zierath JR, Sweeney G (2002) Analysis of paradoxical observations on the association between leptin and insulin resistance. FASEB J 16:1163-1176

50. Cavarape A, Feletto F, Mercuri F, Quagliaro L, Daman G, Ceriello A (2001) High-fructose diet decreases catalase mRNA levels in rat tissues. J Endocrinol Invest 24:838-845 